\title{
Lateral Infrared Photovoltaic Effects in Ag-Doped ZnO Thin Films
}

\author{
Wenwei Liu, ${ }^{1,2}$ Songqing Zhao, ${ }^{2}$ Kun Zhao, ${ }^{1,2,3}$ and Wei Sun ${ }^{2}$ \\ ${ }^{1}$ State Key Laboratory of Petroleum Resource and Prospecting, China University of Petroleum, Beijing 102249, China \\ ${ }^{2}$ Laboratory of Optic Sensing and Detecting Technology, China University of Petroleum, Beijing 102249, China \\ ${ }^{3}$ International Center for Materials Physics, Chinese Academy of Sciences, Shenyang 110016, China
}

Correspondence should be addressed to Kun Zhao, zhk@cup.edu.cn

Received 18 November 2009; Revised 12 April 2010; Accepted 16 April 2010

Academic Editor: Mario Pagliaro

Copyright () 2010 Wenwei Liu et al. This is an open access article distributed under the Creative Commons Attribution License, which permits unrestricted use, distribution, and reproduction in any medium, provided the original work is properly cited.

\begin{abstract}
A transient lateral photovoltaic effect has been observed in Ag-doped $\mathrm{ZnO}$ thin films. Under the nonuniform irradiation of a $1064 \mathrm{~nm}$ pulsed laser, the photovoltaic response shows high sensitivity to the spot position on the film surface. The highest photovoltaic responsivity of $27.1 \mathrm{mV} \mathrm{mJ}^{-1}$ was observed, with a decline time of $\sim 1.5 \mathrm{~ns}$ and a full width at half-maximum (FWHM) of $\sim 4 \mathrm{~ns}$. The photovoltaic position sensitivity can reach about $3.8 \mathrm{mV} \mathrm{mJ}^{-1} \mathrm{~mm}^{-1}$. This paper demonstrates the potential of Ag-doped $\mathrm{ZnO}$ films in the position-sensitive infrared detection
\end{abstract}

\section{Introduction}

$\mathrm{ZnO}$ is of technological importance and considerable scientific interest due to its unique properties such as direct wide band gap, large exciton, binding energy, strong emission, large saturation velocity $\left(3.2 \times 10^{7} \mathrm{~cm} \mathrm{~s}^{-1}\right)$, high radiation resistance, and high breakdown voltage [1]. Therefore, $\mathrm{ZnO}$ is a promising candiate for high-power and high-frequency semiconductor devices such as ultraviolet (UV) detectors and laser devices [2-4]. Modification of $\mathrm{ZnO}$ properties by impurity incorporation is currently another important issue for possible applications in UV optoelectronics. Doping in $\mathrm{ZnO}$ with selective elements offers an effective method to adjust their electrical, optical, and magnetic properties, which is crucial for the practical applications. For example, $\mathrm{Al}$ doping in $\mathrm{ZnO}$ increased its conductivity without impairing the optical transmission, B doping decreased the resistivity [5], Mn-doped $\mathrm{ZnO}$ had ferromagnetism [6], and $\mathrm{Ga}_{2} \mathrm{O}_{3}$ in $\mathrm{ZnO}$ enhanced the near band edge UV emission [7], sulfur doping decreased dramatically the UV emission intensity [8]. The merits of wide band gap and large exciton binding energy of $\mathrm{ZnO}$ which lead to the research attentions, and Schottky contact, and p-n junction type of $\mathrm{ZnO}$-based UV photodetectors have been realized and reported [9-11]. However, less attention has been paid to the infrared characteristics in $\mathrm{ZnO}$. Following up with our previous work, here, we show that Ag-doped $\mathrm{ZnO}$ thin film exhibits a lateral laser-induced photovoltage: the saturation values of this photovoltage vary with the the position of the laser spot under illumination with the $1064 \mathrm{~nm}-\mathrm{Nd}$ :YAGpulsed infrared laser. The lateral photovoltaic property is expected to make the $\mathrm{ZnO}$ a candidate for position-sensitive photodetectors, and the possible mechanisms are addressed.

\section{Experimental}

In our paper the silver-doped $\mathrm{ZnO}$ thin film was fabricated on fused quartz substrates $(10 \mathrm{~mm} \times 10 \mathrm{~mm})$ which was prepared from a $\mathrm{ZnO}$ mosaic target (1/4 area of the target was uniformly covered with high purity of silver slice in the shape of sector) on fused quartz substrates by pulsed laser deposition (PLD) [12] using a $\mathrm{KrF}$ excimer laser with the wavelength $248 \mathrm{~nm}$, pulse duration $30 \mathrm{~ms}$, energy density $1 \mathrm{~J} / \mathrm{cm}^{2}$ in $\mathrm{O}_{2}$ atmosphere with the pressure $5 \times 10^{-4} \mathrm{~Pa}$ and the substrate growth temperature is $450^{\circ} \mathrm{C}$. The films thickness was controlled by the number of laser pulses at $450^{\circ} \mathrm{C}$ by pulsed-laser deposition. The SEM morphology of Ag-doped $\mathrm{ZnO}$ films deposited at substrate temperature of $450^{\circ} \mathrm{C}$ was reported in [13]. The film surfaces are very 


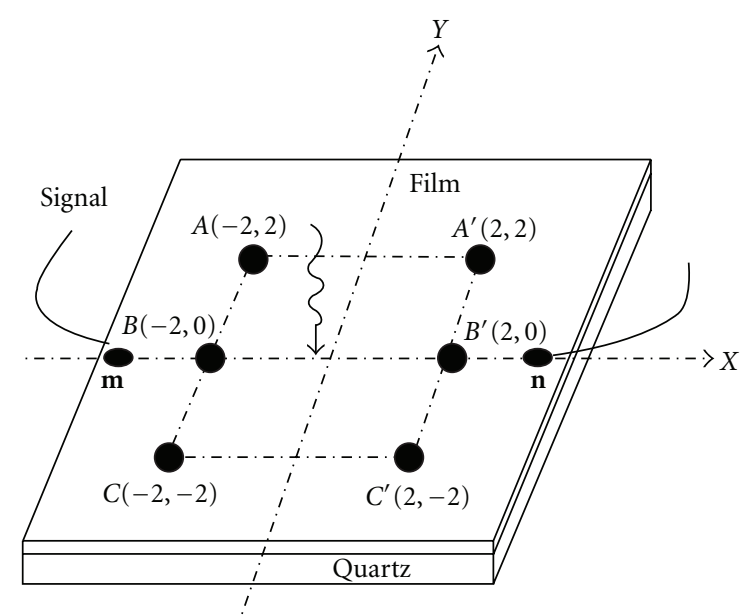

Figure 1: The schematic diagram displays the electrode settings, the laser spot, and the laser spot moving direction.

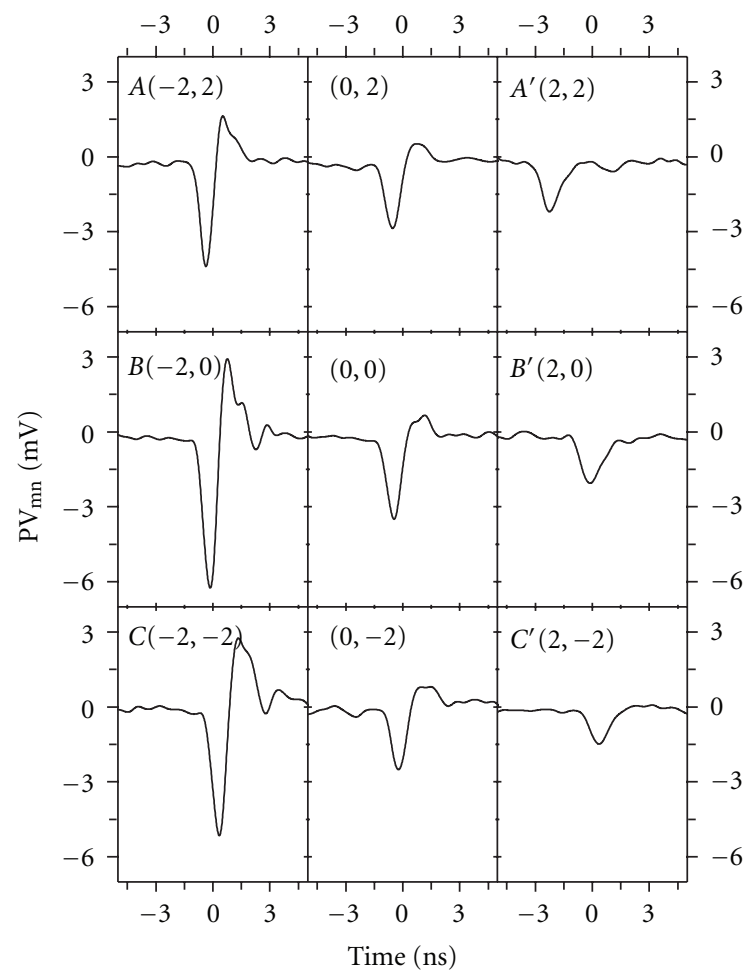

Figure 2: Photovoltaic signals $\mathrm{PV}_{\mathrm{mn}}$ between $\mathbf{m}$ and $\mathbf{n}$ electrodes under the irradiation of $1064 \mathrm{~nm}$ pulse laser at selected positions of $(-2,2),(0,2),(2,2),(-2,-2),(0,-2)$, and $(2,-2)$, respectively.

smooth, basically silver nanocluster uniformly distributed in the film.

For the measurment of optoelectric behaviors, the $1 \mathrm{~mm}$ diameter indium electodes were made on the film surface. The $1064 \mathrm{~nm}$ infrared Nd:YAG laser (pulse duration of $25 \mathrm{ps}$, repetition rate of $10 \mathrm{~Hz}$ ) was used as the light source at ambient temperature in air with a pulse energy of $0.23 \mathrm{~mJ}$ and a light spot of $2 \mathrm{~mm}$ in diameter locked by a diaphragm.

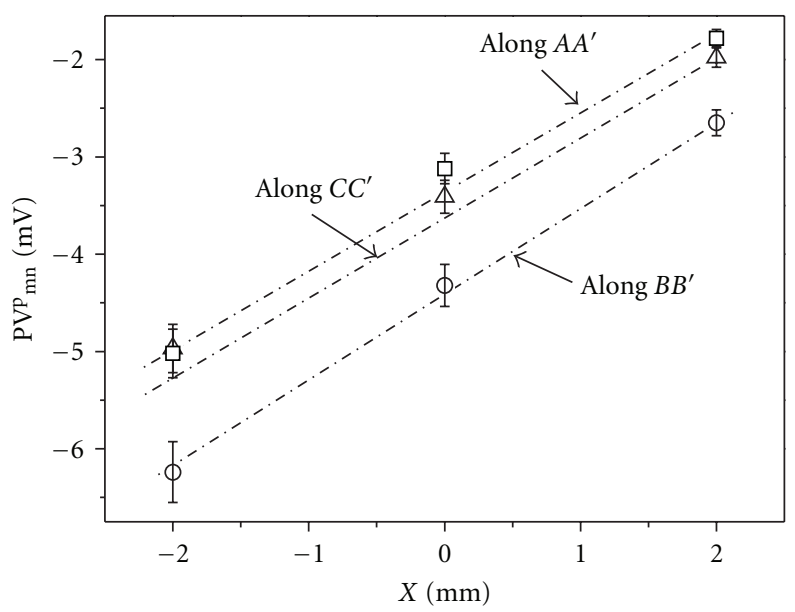

Figure 3: Dependence of the $\mathrm{PV}^{\mathrm{p}}{ }_{\mathrm{mn}}$ on the position of the laser spot in the $x$ direction when laser is irradiated on the $\mathrm{ZnO}$ surface.

As shown in Figure 1, the laser irradiated the sample along $A A^{\prime}, B B^{\prime}$, and $C C^{\prime}$ directions, and the photovoltaic signals from the anode $(\mathbf{m})$ to cathode $(\mathbf{n})$ were monitored with a sampling oscilloscope terminated into $50 \Omega$. The square $\left(A C C^{\prime} A^{\prime}\right)$ region as displayed in Figure 1 is chosen in order to avoid the effects from the light illumination on the edge and electrodes.

\section{Result and Discussion}

Initial photoconductive infrared (IR) detectors have been developed utilizing band-band transitions or dopant-toband transitions. Here, we have the $\mathrm{Ag}$ as a dopant in $\mathrm{ZnO}$, and we make it exposed to IR laser. Figure 2 showed the photovoltaic responses to the $1064 \mathrm{~nm}$-pulsed laser irradiation varying with the laser spot along the $A A^{\prime}$, $B B^{\prime}$, and CC' directions. Two significant characteristics of the photovoltaic signals were found: (i) the maximum photovoltage is obtained when the laser spot is very close to the $\mathbf{m}$ electrode; (ii) moving the laser spot between the $\mathbf{m}, \mathbf{n}$ electrodes leads to the photovoltage dropping. The highest photovoltaic responsivity of $27.1 \mathrm{mV} \mathrm{m}^{-1}$ was observed, with a decline time of $\sim 1.5 \mathrm{~ns}$ and a full width at half-maximum (FWHM) of $\sim 4 \mathrm{ns,} \mathrm{along} \mathrm{the} \mathrm{BB}^{\prime}$ direction close to the $\mathbf{m}$ electrode. The $x$ direction-position sensitivity, which means the variation of the photovoltage in $\mathrm{mV} \mathrm{mJ}^{-1}$ for a $1 \mathrm{~mm}$ displacement of the spot along $x$ direction, is about $3.8 \mathrm{mV} \mathrm{mJ}^{-1} \mathrm{~mm}^{-1}$.

As displayed in Figure 3, the lateral photovoltage was obtained through two indium electrodes named $\mathbf{m}$ and $\mathbf{n}$ located in the middle of the two opposite film sides. The peak values of the laser induced photovoltages $\left(\mathrm{PV}^{\mathrm{p}}{ }_{\mathrm{mn}}\right)$ were plotted as a function of the laser spot position $x$ (along $A A^{\prime}$, $B B^{\prime}$, and $C C^{\prime}$, the coordinate origin $\mathrm{O}$ was set at the centre between $B$ and $B^{\prime}$ ) on the $\mathrm{ZnO}$ surface. In the region between the electrodes along the $x$-axis (shown in Figure 1), when the laser irradiated on the $\mathrm{ZnO}$ surface, the $\mathrm{PV}^{\mathrm{p}}{ }_{\mathrm{mn}}$ varied very linearly with laser position $x$. It is clear that the absolute 


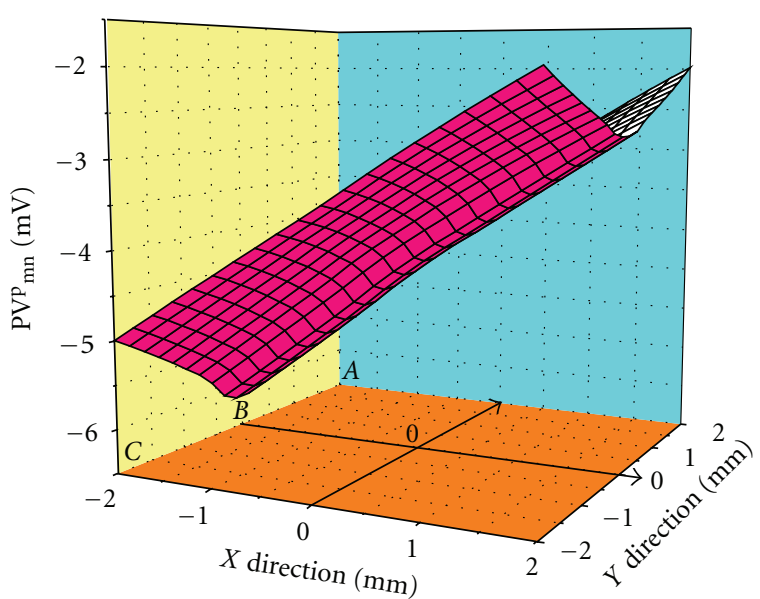

FIgURE 4: Three-dimensional plot for the $\mathrm{PV}^{\mathrm{p}}{ }_{\mathrm{mn}}$ as a function of the laser spot position.

$\mathrm{PV}^{\mathrm{p}}{ }_{\mathrm{mn}}$ value depends on the position of the spot on the $x$ axis and undergoes a sign drop while the laser spot travels from $\mathbf{m}$ to $\mathbf{n}$. The biggest absolute signal value occur when the light spot is close to $B$.

Figure 4 summarizes the spatial distribution of the $\mathrm{PV}_{\mathrm{mn}}^{\mathrm{p}}$. The schemae looks like an open book with the ridge toward $X-Y$ plane. The photovoltaic signals are almost symmetrical. The dependence of the photovoltage of laser spot position shows a higher value for the laser spot close to the $\mathbf{m}$ electrode while a lower value close to the $\mathbf{n}$ electrode. The result indicated the potential in positionsensitive detection at room temperature.

Ag-doped $\mathrm{ZnO}$ thin film exhibits a sharp absorption edge at $370 \mathrm{~nm}$ [12] in agreement with its band gap of $3.37 \mathrm{eV}$ and photocarrier can be easily generated under UV light illumination. However, the photon energy of $1064 \mathrm{~nm}$ laser is $1.165 \mathrm{eV}$, and it is impossible for the $1064 \mathrm{~nm}$ photons to excite the electron-hole pairs in either the Ag-doped $\mathrm{ZnO}$ thin film or the insulating quartz substrate. The above fact demonstrates the other aspects playing a crucial role in the photoresponse process in the present sample.

The lattice mismatch between the $\mathrm{ZnO}$ and quartz (major component is $\mathrm{SiO}_{2}$ and high concentration traps exist resulting from impurities or defects) lead to the Ag doping on the fused quartz as surface plasmon at the interface which plays the role of capping buffer layer. So, many microdomains originated from the interface between the film and substrate and even run through the thickness of the whole film, resulting into nanorods, nanowires, and nanoclusters [13]. Thus, there are many metal-insulator (MI) contact structures in Ag-doped $\mathrm{ZnO} /$ quartz film. Based on MI contact theory [14], the Fermi level (Ef) of Ag immediately after contact is higher than that of $\mathrm{SiO}_{2}$, and electrons will be transferred from the $\mathrm{Ag}$ into $\mathrm{SiO}_{2}$ to equalize the Ef. During the transmission, a portion of electrons are trapped by positively charged centres in the crystal and are accumulated in traps as discussed by Terner [14] and Liu et al. [15]. The contact potential difference in the equilibrium state is estimated as $\sim 0.64 \mathrm{eV}$ being determined by the metal and insulator work functions, which is smaller than the $1064 \mathrm{~nm}$ photon energy of $1.165 \mathrm{eV}$. Under $1064 \mathrm{~nm}$ laser irradiation, a number of trapped electrons are freed into the conduction band of $\mathrm{SiO}_{2}$ and removed by the built-in electric field. Eventually, the photovoltages were induced. Further experiments, such as polarization, doping concentration, interface, and tilting angle dependences, are in progress in order to clarify the underlying detection mechanism of the Ag-doped $\mathrm{ZnO}$ thin film.

\section{Conclusions}

In summary, we have observed the lateral photovoltaic effects in the Ag-doped $\mathrm{ZnO}$ thin film under the irradiation of the $1064 \mathrm{~nm}$ pulse laser. The largest photovoltaic responsivity is $\sim 27.1 \mathrm{mV} \mathrm{mJ}^{-1}$ with the decline time of $\sim 1.5 \mathrm{~ns}$ and a FWHM of $\sim 4 \mathrm{~ns}$. The peak photovoltage shows a high sensitivity of laser spot position between the contacts on the film surface. In addition, avoiding cryogenic cooling not only reduces the cost and weight but also simplifies the infrared detector system, allowing widespread usage. $\mathrm{ZnO}$ is a feasible solution to the future of uncooled IR detection, and the promising initial results demonstrate the possiblity of Agdoping $\mathrm{ZnO}$ as a candidate of IR position detector.

\section{Acknowledgment}

This paper has been supported by NCET, NSFC, RFDP, and Beijng Natural Science Foundation.

\section{References}

[1] C. E. Johnson, W. A. Weimer, and D. C. Harris, "Characterization of diamond films by thermogravimetric analysis and infrared spectroscopy," Materials Research Bulletin, vol. 24, no. 9, pp. 1127-1134, 1989.

[2] K. W. Liu, J. G. Ma, J. Y. Zhang, et al., "Ultraviolet photoconductive detector with high visible rejection and fast photoresponse based on $\mathrm{ZnO}$ thin film," Solid-State Electronics, vol. 51, no. 5, pp. 757-761, 2007.

[3] S. Chu, M. Olmedo, Z. Yang, J. Kong, and J. Liu, "Electrically pumped ultraviolet $\mathrm{ZnO}$ diode lasers on Si," Applied Physics Letters, vol. 93, no. 18, Article ID 181106, p. 3, 2008.

[4] D.-K. Hwang, M.-S. Oh, J.-H. Lim, Y.-S. Choi, and S.-J. Park, "ZnO-based light-emitting metal-insulator-semiconductor diodes," Applied Physics Letters, vol. 91, no. 12, Article ID 121113, 3 pages, 2007.

[5] B. J. Lokhande, P. S. Patil, and M. D. Uplane, "Studies on structural, optical and electrical properties of boron doped zinc oxide films prepared by spray pyrolysis technique," Physica B, vol. 302-303, pp. 59-63, 2001.

[6] P. Sharma, A. Gupta, K. V. Rao, et al., "Ferromagnetism above room temperature in bulk and transparent thin films of Mndoped ZnO," Nature Materials, vol. 2, no. 10, pp. 673-677, 2003.

[7] R. Al Asmar, S. Juillaguet, M. Ramonda, et al., "Fabrication and characterization of high quality undoped and $\mathrm{Ga}_{2} \mathrm{O}_{3}$-doped $\mathrm{ZnO}$ thin films by reactive electron beam coevaporation technique," Journal of Crystal Growth, vol. 275, no. 3-4, pp. 512-520, 2005. 
[8] G. Shen, J. H. Cho, J. K. Yoo, G.-C. Yi, and C. J. Lee, "Synthesis and optical properties of S-doped $\mathrm{ZnO}$ nanostructures: nanonails and nanowires," Journal of Physical Chemistry B, vol. 109, no. 12, pp. 5491-5496, 2005.

[9] S. Liang, H. Sheng, Y. Liu, Z. Huo, Y. Lu, and H. Shen, "ZnO Schottky ultraviolet photodetectors," Journal of Crystal Growth, vol. 225, no. 2-4, pp. 110-113, 2001.

[10] T. K. Lin, S. J. Chang, Y. K. Su, B. R. Huang, M. Fujita, and Y. Horikoshi, "ZnO MSM photodetectors with $\mathrm{Ru}$ contact electrodes," Journal of Crystal Growth, vol. 281, no. 2-4, pp. 513-517, 2005.

[11] J. L. Liu, F. X. Xiu, L. J. Mandalapu, and Z. Yang, "P-type ZnO by Sb doping for PN-junction photodetectors," in Zinc Oxide Materials and Devices, vol. 6122 of Proceedings of the SPIE, January 2006.

[12] W. Liu, S. Zhao, K. Zhao, et al., "Ultraviolet photovoltaic characteristics of silver nanocluster doped $\mathrm{ZnO}$ thin films," Physica B, vol. 404, no. 8-11, pp. 1550-1552, 2009.

[13] S. Zhao, Y. Zhou, K. Zhao, et al., "Violet luminescence emitted from Ag-nanocluster doped $\mathrm{ZnO}$ thin films grown on fused quartz substrates by pulsed laser deposition," Physica B, vol. 373, no. 1, pp. 154-156, 2006.

[14] W. J. Turner, "Photoemission from silver into sodium chloride, thallium chloride, and thallium bromide," Physical Review, vol. 101, no. 6, pp. 1653-1660, 1956.

[15] H. Liu, K. Zhao, S. Zhao, et al., "Ultrafast and spectrally broadband photovoltaic response in quartz single crystals," Journal of Physics D, vol. 42, no. 7, Article ID 075104, 2009. 


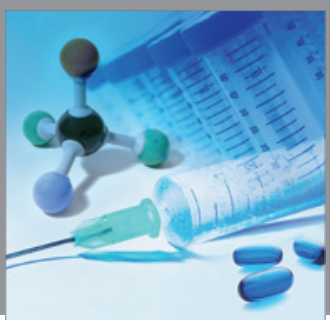

International Journal of

Medicinal Chemistry

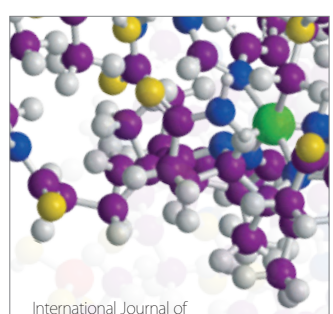

Carbohydrate Chemistry

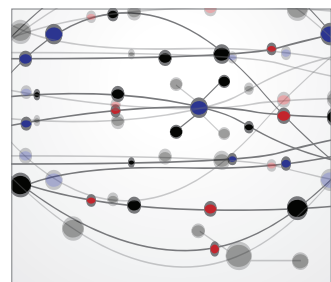

The Scientific World Journal
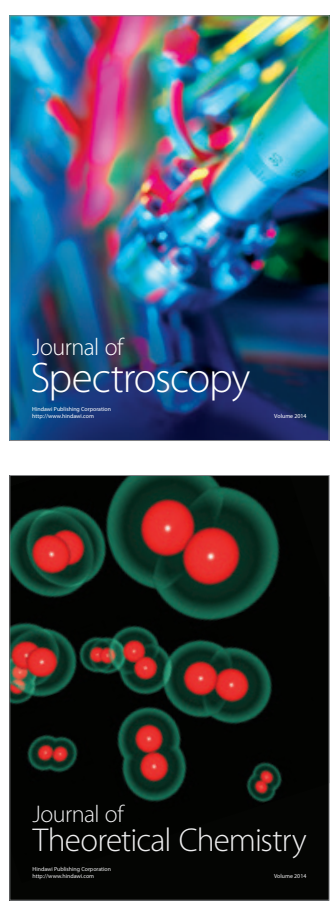
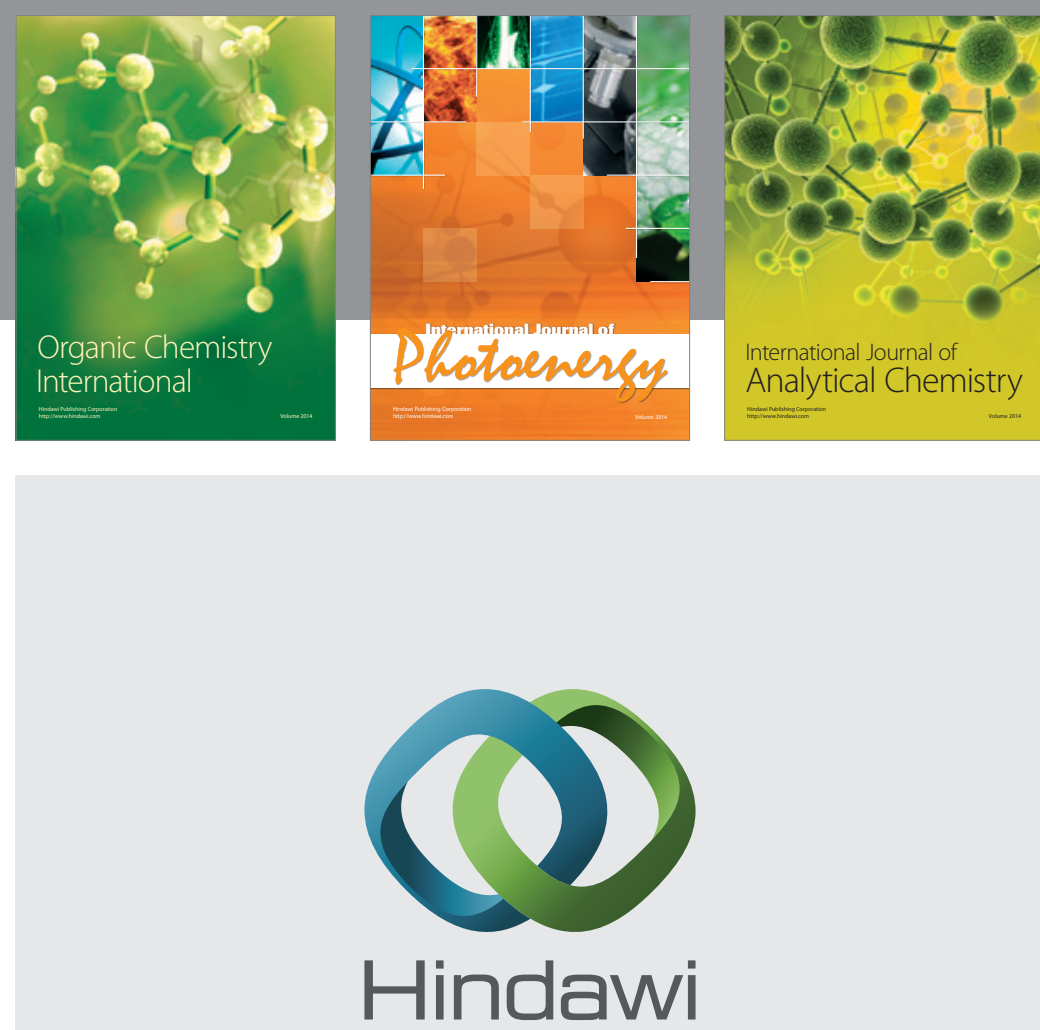

Submit your manuscripts at

http://www.hindawi.com
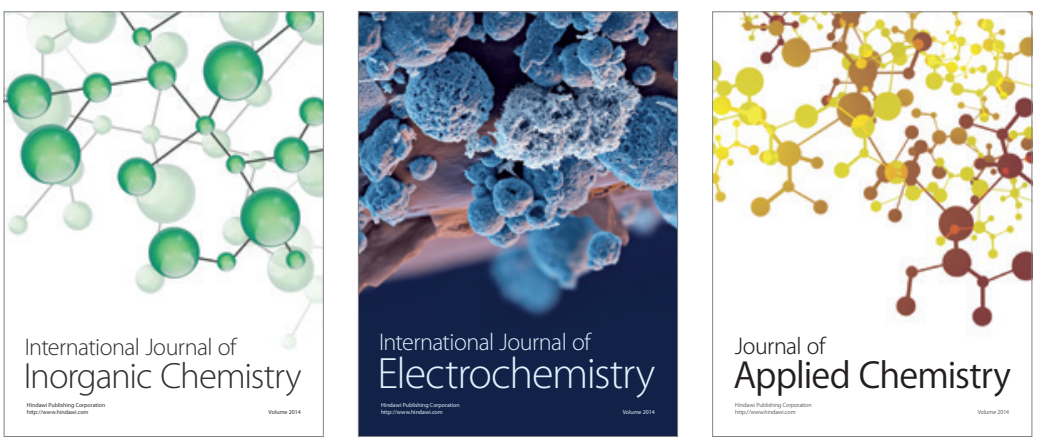

Journal of

Applied Chemistry
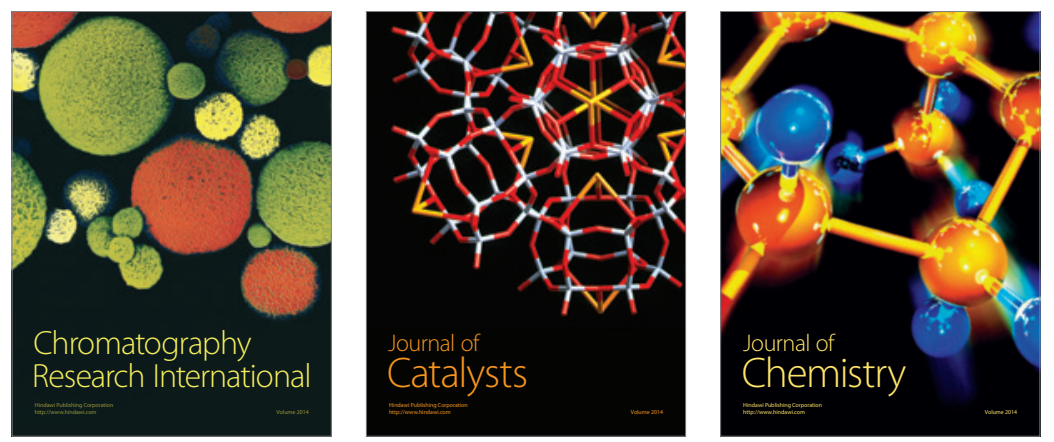
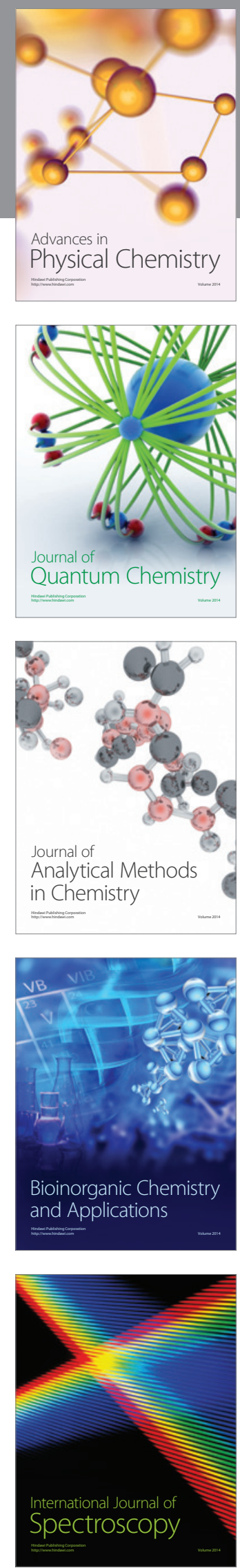